\title{
Toxicology of environmentally persistent chlorinated organic compounds
}

\author{
Annika Hanberg \\ Institute of Environmental Medicine, Karolinska Institutet, Box 210, S-171 77 Stockholm, \\ Sweden
}

Abstract: The chlorinated organic compounds comprise a large group of compounds with a complex diversity of biologic effects and mechanisms of action. Based on the persistence and high toxicity of dioxins, PCBs, and DDT, this chapter focuses primarily on the toxicology of these organochlorine compounds. Not even these groups of compounds can be regarded as homogenous since the individual congeners show diverse environmental fates and toxicities. In this chapter, primarily the critical effects suspected to occur at human exposure at background levels are discussed, i.e. cancer, and effects on reproduction, neurobehaviour and the immune system. In recent years, interactions between these compounds and hormonal systems have received increasing attention. They have also been shown to be particularly toxic to the developing embryo, fetus and infant. In order to conduct risk assessment with a higher degree of accuracy, there is a need for further mechanistical data, and additional epidemiological studies, especially with regard to effects on reproductive, neurobehavioural and immune systems. The data presently available suggest that such effects already occur in the human population and thus, in order to reduce the exposure to these compounds, further release into the environment should be prevented.

\section{INTRODUCTION}

The chlorinated organic compounds comprise a large group of compounds with a complex diversity of biologic effects. However, this fact has not always been a prominent feature in the public debate. This chapter will summarize the toxicology of some organochlorine compounds, selected on the basis of their general occurrence as environmental pollutants and available information on their toxicity. The compounds selected are the dioxins (PCDDs and PCDFs), PCBs, DDT, as well as some other organochlorine pesticides. There are, however, a variety of other chlorinated organic compounds, in fact the largest part of this group, which either are not considered toxic to humans at the levels occurring in the environment or which are not yet sufficiently studied with regards to their occurrence and/or toxic effects.

The dioxins, PCBs, DDT and the organochlorine pesticides have several properties in common which make them toxicologically important. First, they are generally very resistant to both chemical and biological degradation and they are highly lipophilic. Consequently, they accumulate in food chains and human exposure occurs primarily via food. Due to their occurrence as environmental pollutants and their common properties, the organochlorines tend to be found together [1]. The composition of the mixtures of lipophilic pollutants found in human tissues is relatively similar, but will vary according to regional differences in use and pollution of the various compounds, as well as the dietary habits of different populations. For example, in Sweden, fish is a dominating source of exposure due to the comparatively high intake of fish, while in the Netherlands the intake of dairy products is the most important source of exposure. In addition to local discharge, the chlorinated organic pollutants are globally spread as they are transported in the atmosphere from warmer areas and condensate when they reach cooler areas, such as the Great lakes or the Arctic region.

This chapter is dedicated to the memory of Professor Ulf G. Ahlborg, a leading expert in the field of toxicology and risk assessment of chlorinated organic compounds, and my professor and personal friend, who very sadly and much too early died in January 1996. 
Blood levels reflect adipose tissue stores of the lipophilic organochlorine contaminants. Although estimated rates of elimination vary, with few exceptions organochlorines are eliminated slowly from the body. Among women, lactation is the most important route of excretion for these organochlorines [2]. Consequently, the breast-fed infant is highly exposed to these compounds. For most of these chemicals the body burden increase with increasing age. This is most probably the consequence of both accumulated storage in the body with time, and the fact that older individuals have been exposed to higher levels of these compounds. Because of their widespread occurrence in the environment and their high toxicity, especially dioxins and PCBs have over the last decades become the subject of intense public and scientific concern. In recent years, the public debate has also focused on the interference of certain organochlorine compounds with hormonal systems, primarily the estrogenic system. Due to a high exposure and/or a probable high sensitivity, the human fetus and breast-fed infant are regarded as the primary risk groups of these compounds [3-5]. The present chapter will primarily focus on the critical effects suspected to occur at human exposure at background levels, i.e. cancer, and effects on reproduction, neurobehaviour and the immune system. In particular, effects induced in the embryo, fetus and infant will be discussed. In addition, some proposed mechanisms of action to cause these effects will be described.

\section{PERSISTENT CHLORINATED ORGANIC COMPOUNDS}

\section{Dioxins and dioxin-like compounds}

2,3,7,8-Tetrachlorodibenzo-p-dioxin (TCDD), the most toxic dioxin congener, is in toxicology used as a prototype for all dioxin-like compounds, i.e. compounds structurally and toxicologically similar to TCDD. The dioxin-like PCDDs and PCDFs are chlorinated in the 2-, 3-, 7- and 8-positions of the molecule and their toxic potency decreases with an increasing number of further chlorination. Also for the PCBs, the congeners chlorinated only in the lateral parts of the molecule (i.e. the non-ortho- or planar congeners) show the highest dioxin-like toxicity.

The dioxin-like compounds elicit a broad spectrum of responses that are specific for the age, sex, strain, and species of the animal studied [reviewed by refs 3,6-9]. Among these effects are decreased food consumption, a wasting syndrome, hepatotoxicity, thymic and lymphoid involution accompanied by diverse immuno-suppressive effects, carcinogenesis, teratogenicity, developmental and reproductive toxicity, dermal toxicity, diverse effects on hormones and growth factors, and induction of phase I and phase II drugmetabolizing enzyme activities. Toxicity in humans exposed to dioxin-like compounds has primarily been described after accidental or occupational exposure [for review see refs 3,6]. The effects reported in humans include primarily dermal symptoms (e.g. chloracne, hyperkeratosis, hyperpigmentation), but also liver fibrosis, hypertri-glyceridemia, loss of appetite and weight loss. The effects of exposure to dioxinlike compounds that have been identified as critical for human exposure, i.e. cancer and effects on reproductive systems, neuro-behaviour and immune function, will be described later in this chapter.

\section{PCBs}

Cancer and effects on behaviour and the immune system have been observed after long-term exposure to relatively low doses of PCB-mixtures (see later in this chapter). Whether dioxin-like or nondioxin-like PCB congeners are responsible for these effects are not yet clear. A further complication with PCBs, in contrast to dioxins, is the formation of persistent and reactive metabolites, i.e. hydroxylated and methylsulfonyl metabolites.

\section{DDT and other organochlorine pesticides}

DDT and many other chlorinated insecticides have been found to produce predominantly liver tumours in rodents. Some of these compounds have also been reported to show some estrogenic effects in cellular or animal systems. However, except for DDT, the toxicological information on the chlorinated insecticides are scarce with regard to these effects. As is the case with the PCBs, a problem involved in the evaluation 
of the toxicology of DDT is the various DDT compounds showing different effects and probably acting through different mechanisms. The most estrogenic form of DDT is $o, p^{\prime}-\mathrm{DDT}$, which constitutes $\approx 15 \%$ of the technical product. This form of DDT is, however, more easily eliminated in animals and humans, while the major metabolite of DDT, found in food, $p, p^{\prime}$-DDE is very stable. Thus, the DDT mixture found in human tissues consists of primarily $p, p^{\prime}$-DDE and to a much lower degree, of $a, p^{\prime}$-DDT [10]. The toxicological data on cancer and estrogenic effects of organochlorine insecticides are described in later sections of this chapter.

\section{Environmental mixtures of persistent chlorinated organic compounds}

In some studies, human populations are studied which are exposed to mixtures of persistent environmental pollutants, via consumption of contaminated food stuffs, such as fish from polluted waters. The effects seen in these studies cannot be clearly associated with any one of the pollutants occurring in these food items or in human tissues, although some compounds are more likely the cause than others.

\section{MECHANISMS OF ACTION}

Chlorinated organic compounds exert their toxicity through several mechanisms. However, action through binding of dioxin-like compounds to the intracellular Ah receptor and/or interference with hormonal systems are considered as relevant mechanisms for the effects occurring at low dose exposure of these chemicals and will be discussed in the following section.

\section{Ah receptor}

The proposed mechanism of action for dioxin-induced effects involves binding of the compound to the intracellular aryl hydrocarbon receptor $(\mathrm{AhR})$ and subsequent regulation of gene expression. It is generally agreed that binding of TCDD to the Ah receptor constitutes a first and necessary step to elicit the toxic and biochemical effects of this compound. It is, however, also clear that this binding is not sufficient to elicit the effects, but subsequent steps of activation and DNA-binding are necessary [for review see ref. 11]. Several recent reviews have described the mechanism of AhR action [12-14]. In the cytoplasm, the unliganded $A h R$ is present in a latent, inactive configuration associated with the $90 \mathrm{kDa}$ heat shock protein (hsp90) which prevents DNA-binding and also keeps the receptor in a conformation that can bind the ligand. Ligand binding induces the loss of hsp90 and translocation of the AhR-ligand complex to the nucleus. When heterodimerized with the Ah receptor nuclear translocator protein (Arnt), the ligand-AhRArnt complex is able to bind to dioxin responsive elements on the DNA and initiate transcription of specific genes. This mechanism of action of TCDD parallels in many ways that of the steroid hormones. However, dioxin and steroid hormone receptors (e.g. estrogen, androgen, glucocorticoid, thyroid, vitamin $\mathrm{D}_{3}$ and retinoic acid receptors) do not belong to the same family since AhR and Arnt interact with DNA through a helix-loop-helix motif rather than the zinc finger motif of the nuclear hormone receptors [14].

The mechanism of dioxin action via the AhR has hitherto been demonstrated only for the TCDDinduced induction of CYP1A1. However, a recent study using AhR null mutant mice have shown that the AhR is involved in the induction also of CYP1A2 and UDPGT [15]. In addition, other genes have been shown to be either directly controlled by the AhR or to be responsive to AhR agonists. Generally, products of these genes are either growth-regulatory proteins or drug-metabolizing enzymes [13]. Most of the existing knowledge on the mechanism of action of TCDD-induced effects other than enzyme induction is, however, largely correlative [16]. A number of the effects elicited by TCDD, such as lethality, thymic atrophy, induction of cleft palate, chloracne, tumour promotion, and enzyme induction, were demonstrated to segregate with the Ah allele $[9,17]$. Mice having the responsive allele (i.e. a high affinity form of AhR) were more sensitive to these effects. In addition, structure-activity relationships demonstrate that for dioxinlike compounds, the toxicity of individual congeners is correlated with the affinity with which the congeners bind to the AhR $[13,18]$. Moreover, several AhR partial antagonists inhibit or reduce the toxic and biochemical effects of TCDD [13]. Most of these correlative studies concerns, however, endpoints such as enzyme induction and effects on organ weights. 


\section{The TEF concept}

Humans are exposed to a multitude of different dioxin-like compounds, whose proposed common mechanism of action (i.e. via the Ah receptor) forms the basis for the current risk assessment of mixtures of these substances by using a toxic equivalency factor (TEF) concept and TCDD as a reference compound $[19,20]$. Thus, the total TCDD-like toxicity of a mixture can be expressed as one value, the toxic equivalent, which can be used to assess the risk for humans to consume for example fish or mother's milk. The TEF-system has, however, several weaknesses [20,21]. The TEF-concept assumes additivity for all dioxin-like compounds in a mixture, a phenomenon which has not been clearly demonstrated. Moreover, the concept assumes identical mechanisms of action and dose-response curves for the critical effects in humans, i.e. effects on reproduction, fetal development, immune function, and tumour development, and for the effects that the TEF system is based upon, e.g. enzyme induction.

\section{Hormonal mechanisms}

The chlorinated organic compounds, covered in this chapter, interfere with several hormonal systems, such as estrogens, thyroid hormones, glucocorticoids and retinoic acid [10,22]. These effects occur at different levels, such as altered metabolism of hormones, altered receptor levels, or interference with transport proteins. In the following section the effects on the sexual hormones, estrogen and androgen, will be briefly discussed.

\section{Antiestrogenicity}

There is considerable evidence that TCDD has antiestrogenic properties. This evidence includes both inhibition of the development of spontaneous mammary tumours in female rats, as well as a diverse spectrum of antiestrogenic responses in rodents and in human breast cancer cell lines [reviewed by ref. 10]. Results from studies with mouse strains and cell lines differing in the functionality of the Ah receptor, as well as from structure-activity studies with different PCDDs, suggest that the antiestrogenic responses of TCDD and related compounds are mediated through the Ah receptor. Although the induced metabolism of $17 \beta-$ estradiol by dioxin-like compounds may cause some of the antiestrogenic effects of TCDD at relatively high doses, the following results do not support that hypothesis: circulating levels of $17 \beta$-estradiol are not affected by TCDD, and down-regulation of the estrogen receptor occurs both earlier and at lower doses than enzyme induction [23]. The antiestrogenic effects are not due to TCDD or estradiol cross-reacting with each other's receptor. Instead, the data suggest that the AhR-ligand complex regulates the synthesis of estrogen receptor mRNA [24].

\section{Estrogenicity}

There is considerable evidence on the estrogenicity of $o, p^{\prime}$-DDT [for review see ref. 10]. Some (perhaps all) of the effects are caused by the binding of $o, p^{\prime}$-DDT to the estrogen receptor. Weak estrogenic activity in some animal studies has also been noted for $p, p^{\prime}$-DDT, the active pesticide. Due to structural similarities to estradiol, hydroxylated PCB metabolites that are also conformationally restricted due to ortho substitution might be effective in binding the estrogen receptor [25]. Consequently, certain PCB congeners, putative PCB metabolites and complex PCB mixtures have been reported to be estrogenic. The organochlorine pesticides, methoxychlor (its demethylated metabolites) and chlordecone, have also been shown to cause one or more estrogenic effects. In addition, estrogenic effects in vitro have been reported for toxaphene, dieldrin and endosulfan [26]. These findings have, however, so far not been verified in vivo. The organochlorine compounds showing estrogenic effects are much less potent (at least 2 orders of magnitude) in this respect than $17 \beta$-estradiol. However, the organochlorine compounds with long biological half-lives accumulate in the food chain and might reach concentrations high enough to elicit significant estrogenic effects.

\section{Antiandrogenicity}

In addition to the estrogenic effects, it has recently been demonstrated that organochlorine environmental pollutants also may act on androgenic hormonal pathways. $p, p^{\prime}$-DDE, the main metabolite of DDT, has been shown to be antiandrogenic [27]. 


\section{CRITICAL EFFECTS FOR HUMAN EXPOSURE}

Effects on tumour development, as well as developmental effects on reproduction, neurobehaviour, and immune function, are proposed to be critical for human exposure to dioxin-like compounds $[3-5,28]$. The fetus and the neonatal offspring of several species have been shown to be particularly sensitive to dioxins and PCBs [5,7,29]. Current risk assessments of dioxin-like compounds are based on the carcinogenic effect of these compounds $[3,19,30]$. Recent concern about dioxins, PCBs and DDT is, however, primarily based on the developmental toxicity [3-5,7,28,29,31].

\section{Cancer}

Several long-term studies have shown that TCDD is a multisite carcinogen, a carcinogen in both sexes, and in several species [reviewed by ref. 32]. Mechanistically, TCDD is a potent tumour promoter with weak or no initiating activity. TCDD-induced tumours in rats are primarily found in females [33], and the sensitivity to hepatic tumours can be modulated by hormones [34]. Thus, interactions of TCDD with hormonally mediated events seem to be a critical component of the carcinogenic mechanisms of TCDD [32]. In addition, TCDD has been shown to inhibit spontaneous mammary tumour development in female rats [33], an effect which agrees well with TCDDs antiestrogenic activities [reviewed by refs 10,35 ].

PCBs have been found to be efficacious tumour promoters in mice and rats [for review see ref. 3]. The ability of $P C B$ mixtures to generate liver tumours appears to be related to the degree of chlorination of the mixture. A recent reevaluation of the liver carcinogenicity studies of PCB mixtures concluded that only the studies with the higher chlorinated mixtures indicated a carcinogenic potency [36]. In addition, a highly chlorinated PCB mixture has been shown to reduce the spontaneous formation of mammary tumours in rats [37]. Different PCB congeners (dioxin-like and nondioxin-like) may cause cancer by different mechanisms they are nevertheless nongenotoxic tumour promoters.

The increase in liver tumour incidence among DDT-treated animals has been confirmed in several investigations [reviewed by ref. 10]. DDT and its structural analogues are all reported to be nongenotoxic. $p, p^{\prime}$-DDT (of unknown purity) has been shown to promote the growth of mammary tumours in male rats [38]. However, given that the apparent promoting effect of DDT was seen in the male rat, and that no evidence of mammary carcinogenesis was seen in female rats and mice fed technical-grade DDT or $p, p^{\prime}$ DDE [39], the relevance of this finding to breast cancer in women is questionable. Also several other chlorinated insecticides have been found to produce predominantly liver cancer in rodents, e.g. methoxychlor, hexachloro-benzene, lindane, hexachlorocyclohexanes, chlordane, heptachlor, heptachlorepoxide, mirex, chlordecone, aldrin and dieldrin.

Although several epidemiological studies on accidental and occupational exposure to dioxins and PCBs show relations to an increased incidence of different tumours, the low quality and/or power of the studies make them difficult to interpret $[40,41]$. Due to their estrogenic properties, several organochlorine compounds have been suggested as responsible for (part of) the increasing trend of human breast cancer [for review see refs 10,42]. Most epidemiological studies are, however, difficult to interpret, mostly because of their small sample size and their failure to adequately control for known breast cancer risk factors. However, there are two nested case-control analyses of cohort data which are larger and better designed studies. One of these studies has been interpreted as supporting an association between $p, p^{\prime}$-DDE, but not PCBs, and breast cancer [43]. However, the larger and better designed study has been interpreted as negative with respect to both exposure types [44]. Thus, it was concluded that an association between the human exposure to environmental levels of organochlorines and an increase in the development of mammary tumours is not supported by these data $[10,42]$. However, results from a recent study on Swedish fishermen's wives support an association between exposure to a mixture of persistent organochlorine compounds through fish consumption and an increased risk for breast cancer [45]. Even if exposure to estrogenic and antiestrogenic compounds in the general population does not causes drastic effects in adults, these compounds might still affect particularly sensitive systems, such as the developing fetus and infant.

\section{Reproductive effects}

Different chlorinated organic compounds have been shown to cause a variety of developmental effects on 
the reproductive system of both male and female animals. A very low prenatal dose of TCDD has been shown to affect the male rat reproductive development. In adult age, spermatogenesis of male offspring was decreased and their sexual behaviour was both demasculinized and feminized [reviewed by ref. 7]. Similar effects on the male reproductive development were also demonstrated by Gray et al. [46], who also reported reproductive alterations in the female offspring, involving structural malformations in the urogenital tract [4]. Cross-fostering studies indicate that the majority of these effects are induced by exposure before birth [47]. PCB mixtures have been shown to affect reproduction and the reproductive systems, e.g. effects on ovulation and the menstrual cycle $[3,10]$. Neonatal exposure to rats has resulted in precocious puberty. The reproductive toxicity of PCBs might be related to the estrogenic and antiestrogenic properties of different PCB congeners (and metabolites thereof). Exposure to $o, p^{\prime}$-DDT mimics the effects of estrogen [for review see ref. 10]. Neonatal exposure to $o, p^{\prime}$-DDT leads to permanent sterility and advanced puberty in female rats. In addition, both $o, p^{\prime}$-DDT and $p, p^{\prime}$-DDT can alter female sexual behaviour. Most data on the reproductive effects of DDT concerns female effects. Also other organochlorine insecticides have been shown to affect reproductive systems [reviewed in ref. 10]; methoxychlor, or rather its demethylated metabolites, and chlordecone are uterotropic in rats.

In contrast to most animal studies, the sparse reports on effects on reproductive systems in humans concerns men and boys. Chlordecone has been reported to cause abnormal spermatogenesis in men accidentally exposed at a production facility [48]. Among the children born to mothers poisoned by PCBs and PCDFs in rice oil in Taiwan (Yu-Cheng), significantly smaller penises have been observed when the boys reach puberty [49].

\section{Behavioural effects}

Developmental effects of organochlorine compounds on the behaviour of the offspring have been observed in rodents, monkeys and humans [for review see refs 3,5]. In Rhesus monkeys, whose mothers were exposed to PCB-mixtures during gestation and lactation, behavioural testing showed retarded learning ability at 6-44 months of age [50,51]. Similarly, effects on cognitive development have also been observed in monkey offspring exposed in utero and via lactation to low levels of TCDD [52]. The possible mechanism through which TCDD and PCB might exert the behavioural effects observed are not yet known. However, mechanisms involving effects on dopamine and thyroid systems have been suggested. In general, cognitive changes produced by in utero exposure to PCBs and dioxins in animals persist throughout adulthood [5].

Developmental effects on the behaviour have also been reported for infants and children. Prenatally exposed Yu-Cheng children showed developmental delays, characterized by lower mean intelligence quotients, still apparent at $8-13$ years of age $[5,53]$. The developmental effects observed in Yu-Cheng children are caused by accidentally high exposure situations. However, these reports show which effects can be expected in humans exposed to high enough levels of PCBs and dioxin-like compounds. In addition, developmental effects on cognitive behaviour have been reported in children whose mothers had eaten relatively large quantities of Lake Michigan fish, contaminated with PCB [54,55]. Prenatal PCB exposure was associated with poorer visual recognition memory at 7 months of age [56] and with poorer performance in the McCarthy test, an IQ-type test, at four years of age [54]. In a study of children from the general population of North Carolina a relation between prenatal PCB exposure and poorer psychomotor performance was shown at 6,12 and 24 months of age [57]. Despite the much larger quantities of PCBs transferred to the infant postnatally via breast feeding, none of the measures of cognitive and motor function in either study related to the level of postnatal PCB exposure. In a recent Dutch study of infants of the general population, the visual recognition memory at 3 or 7 months was not correlated to $\mathrm{PCB}$ or dioxin exposure. The psychomotor scale was negatively correlated with the prenatal PCB exposure at 3, but not at 7 months of age [5]. From this study it was concluded that background in utero and lactational exposure of human infants to levels of dioxins and PCBs in the Netherlands is not related to a serious delay in neurodevelopment [5]. However, levels of PCB and TEQ in mother's milk were negatively correlated with neonatal neurological optimality [58]. A higher percentage of hypotonia was observed in infants exposed to higher levels of planar PCBs in breast milk. 


\section{Immunological effects}

TCDD and related compounds produces a vast number of effects on the immune system, often at very low doses. Both the non-specific and specific, humoral and cell-mediated immune responses are suppressed. Also resistance to infectious challenges is decreased [reviewed by ref. 59]. Thus, the cellular targets of TCDD appear to be multiple. A prominent sign of immunotoxicity in rodents exposed to TCDD is atrophy of the thymus. However, the ability of dioxin to affect for example T-lymphocyte subtype pattern in marmosets and the resistance of mice to influenza viruses, has been shown to occur at doses much lower than those resulting in thymic atrophy. It has been suggested that the developing embryo/fetus may be more sensitive than the adult to immunosuppression induced by TCDD [29] and the effects on the immune system seem to belong to the most sensitive variables affected by TCDD [28]. The relevance for man of subtle effects, such as modifications in the pattern of T-lymphocyte surface receptors is largely unknown. Nevertheless, such changes represent clear-cut biological effects induced by TCDD [29].

There are some reports on immunological effects in humans exposed to dioxins and/or PCBs. Indications of reduced immune response and changes in differentiation pattern of T-lymphocytes were reported in people exposed to TCDD in the Times Beach incident in the US [reviewed by ref. 59]. Besides effects on immunoglobulin and T-lymphocyte numbers in the adult Yu-Cheng population, immunosuppressive effects caused by prenatal exposure to $\mathrm{PCB} / \mathrm{PCDF}$ have been suggested in children as they have elevated incidences of respiratory infections and otitis [60]. In recent years, some studies have been performed also on human populations not accidentally or occupationally exposed to dioxins and PCBs. In a Dutch study of breast-fed and bottle-fed infants from the general population changes in certain immunological parameters were related to exposure to dioxins and/or PCBs [61]. Decreases in the number of monocytes and granulocytes and an increase in the number of cytotoxic T-cells were correlated to TEQ/PCB-levels in breast milk and plasma, respectively. In addition, high consumption of fish from the Baltic sea has been related to an altered differentiation pattern of T-lymphocytes [62]. Moreover, the Inuit population in Arctic Quebec, which has elevated tissue levels of PCBs and dioxins, has a 20-fold higher incidence of infectious disease and otitis in the first year of life than individuals living in the southern Quebec [28].

\section{DISCUSSION AND CONCLUSIONS}

This chapter concerning the toxicology of chlorinated organic compounds has mainly discussed the toxicology of dioxins, PCBs, and DDT. These compounds are known to be persistent and thus able to reach high concentrations both in the environment and in human tissues. In addition, they cause cancer in experimental animals. In recent years, their interactions with hormonal systems have received increasing attention and they have been shown to be particularly toxic to the developing embryo, fetus and infant. However, other chlorinated organic compounds than those described in the present chapter may also contribute to the risk for humans of chlorinated organic compounds. The available toxicological documentation of the other compounds, which constitute the major part of the chlorinated organic compounds, is however, at present not sufficient enough for an evaluation of their contribution to the toxic effects of these group of compounds. It is important to note that the chlorinated organic compounds constitutes a wide group of compounds which cannot be regarded as a homogenous group since the individual compounds, even within the groups of dioxins, PCBs and DDT, show diverse environmental fates and toxicities.

The risk assessments of dioxins and PCBs have hitherto been based on the carcinogenicity of these compounds. However, recent studies show that the developing fetus and infant are extremely sensitive to exposure for these compounds, and effects on behaviour, and the reproductive and immune systems have been reported in both experimental animals as well as in humans. Although the exposure to experimental animals generally is higher than the human exposure, the life-long exposure combined with the exceptional long half-life in humans may result in a considerable human body burden. Consequently, the lowest levels causing developmental neuro-behavioural and reproductive effects in experimental animals, are within the range of current background human body burdens. Effects on neurobehavioural development and immune function have been suggested to occur in human infants and children exposed to background levels of dioxins and PCBs. The neuro-behavioural effects are relatively subtle and although they may not be detectable in the individual child, they may have implications at a population level.

The general population are exposed to these organochlorine pollutants primarily via food, such as fish 
and milk products. The breast-fed infant is exposed to high levels which generally exceed the tolerably daily intake of dioxin-like compounds. However, only few effects on neurodevelopment can be associated to lactational exposure, but appear to be caused prenatally. In addition, breast feeding per se appears to be beneficial for neurodevelopment. Therefore, the benefits of breast feeding appear to outweigh the risks associated with the presence of background levels of PCBs and dioxins in the milk.

It is, however, important to realize that humans are exposed to mixtures of these compounds which may interact, either additively, synergistically or antagonistically. In this aspect, both compounds acting through the same mechanism and compounds acting through different mechanisms, but on the same systems, must be considered in the risk assessment of these organochlorine compounds. The risk assessments of PCBs and DDT are complicated by the various mechanisms of toxicity occurring within these groups of compounds. For example, the exclusive use of the TEF approach may underestimate the risk of functional developmental effects by PCBs, since both AhR-dependent and -independent mechanisms may be involved [5]. In addition, there are other mechanisms than those discussed in the present chapter, which may be relevant for human low dose exposure to the persistent organochlorine compounds. For example, altered levels of dopamine and other neurotransmitters may be important for the PCB-induced neurobehavioural effects. Moreover, the development of the reproductive, nervous and immune systems are all sensitive to hormonal alterations, i.e. both the sexual hormones, as well as other hormones, such as thyroid hormone, glucocorticoids and retinoic acid [22].

Further mechanistical and epidemiological studies are needed to assess the risk for humans exposed to persistent organochlorines, primarily dioxins, PCBs and DDT. Research should focus on the developmental effects on reproductive systems, neurobehaviour and immune function. Mechanistic data linking these effects to certain mechanisms of action, e.g. through the Ah receptor, are necessary for accurate risk assessment of these compounds. Such data are also important for interpreting the validity of the TEF-concept used in risk management of the dioxin-like compounds.

Although the use of PCB and DDT is banned in most Western countries, these compounds still imply problems in handling and destruction of PCB-containing wastes, and since DDT still is being used in some developing countries. To reduce the human exposure of these persistent and toxic compounds effort should be made to reduce further release of these compounds into the environment.

\section{REFERENCES}

1 F. W. Kutz, P. H. Wood, D. P. Bottimore, Rev. Environ. Contam. Toxicol. 120, 1 (1991).

2 A. A. Jensen, Chemical contaminants in human milk, A.A. Jensen, S.A. Slorach, Eds. (CRC Press, 1991 ), p. 9.

3 U. G. Ahlborg, A. Hanberg, K. Kenne, Risk assessment of polychlorinated biphenyls. Nord 1992:26 (Nordic Council of Ministers, Copenhagen, 1992).

4 L. S. Birnbaum, Environ. Health Perspect. 103, 89 (1995).

5 A. Brouwer, U. G. Ahlborg, M. van den Berg, L. S. Birnbaum, E. R. Boersma, B. Bosveld, M. S. Denison, L. E. Gray, L. Hagmar, E. Holene, M. Huisman, S. W. Jacobson, J. L. Jacobson, C. Koopman-Essenboom, J. G. Koppe, B. M. Kulig, D. C. Morse, G. Muckle, R. E. Peterson, P. J. J. Sauer, R. F. Seegal, A. E. Smits-van Prooije, B. C. L. Touwen, N. Weisglas-Kuperus, G. Winneke, Eur. J. Pharmacol. 293, 1 (1995).

6 U. G. Ahlborg, B. Holmstedt, H. Håkansson, C. Rappe, IPCS Environment Health Criteria 88: Polychlorinated dibenzo-para-dioxins and dibenzofurans (World Health Organisation, Geneva, 1989).

7 R. E. Peterson, H. M. Theobald, G. L. Kimmel, Crit. Rev. Toxicol. 23, 283 (1993).

8 R. Pohjanvirta, J. Tuomisto, Pharmacological Reviews 46, 483 (1994).

9 A. Poland, J. C. Knutson, Annu. Rev. Pharmacol. Toxicol. 22, 517 (1982).

10 U. G. Ahlborg, L. Lipworth, L. Titus-Ernstoff, C. C. Hsieh, A. Hanberg, J. Baron, D. Trichopoulos, H. O. Adami, Crit. Rev. Toxicol. 25, 463 (1995).

11 L. S. Birnbaum, Environ. Health Perspect. 102, 157 (1994).

12 O. Hankinson, Annu. Rev. Pharmacol. Toxicol. 35, 307 (1995).

13 A. B. Okey, D. S. Riddick, P. A. Harper, TiPS 15, 226 (1994).

14 L. Poellinger, Inducible gene expression, P.A. Baeuerle, Ed. (Birkhäuser, Boston, 1995), p. 177.

15 P. Fernandez-Salguero, T. Pineau, D. M. Hilbert, T. McPhail, S. S. T. Lee, S. Kimura, D. W. Nebert, S. Rudikoff, J. M. Ward, F. J. Gonzalez, Science 268, 722 (1995).

16 G. Clark, A. Tritscher, D. Bell, G. Lucier, Environ. Health Perspect. 98, 125 (1992).

17 A. Poland, E. Glover, Mol. Pharmacol. 17, 86 (1980).

18 S. Safe, Crit. Rev. Toxicol. 21, 51 (1990).

19 U. G. Ahlborg, Chemosphere 19, 603 (1989). 
20 U. G. Ahlborg, G. C. Becking, L. S. Birnbaum, A. Brower, H. J. G. M. Derks, M. Feeley, G. Golor, A. Hanberg, J. C. Larsen, A. K. D. Liem, S. H. Safe, C. Schlatter, F. Wærn, M. Younes, E. Yrjänheikki, Chemosphere 28, 1049 (1994).

21 U. G. Ahlborg, A. Brouwer, M. A. Fingerhut, J. L. Jacobson, S. W. Jacobson, S. W. Kennedy, A. A. F. Kettrup, J. H. Koeman, H. Poiger, C. Rappe, S. H. Safe, R. F. Seegal, J. Tuomisto, M. van den Berg, Eur. J. Pharmacol. 228, 179 (1992).

22 S. H. Safe, Pharmacology and Therapeutics 67, 247 (1995).

23 S. Safe, M. Harris, L. Biegel, T. Zacharewski, Banbury Report 35. Biological basis for risk assessment of dioxins and related compounds, M.A. Gallo, R.J. Scheuplein, K.A. van der Heijden, Eds. (Cold Spring Harbor Laboratory Press, New York, 1991), p. 367.

24 T. E. K. White, T. A. Gasiewicz, Biochem. Biophys. Res Commun. 193, 956 (1993).

25 J. D. McKinney, C. L. Waller, Environ. Health Perspect. 102, 290 (1994).

26 A. M. Soto, K. L. Chung, C. Sonnenschein, Environ. Health Perspect. 102, 380 (1994).

27 W. R. Kelce, C. R. Stone, S. C. Laws, L. E. Gray, J. A. Kemppainen, E. M. Wilson, Nature 375, 581 (1995).

28 L. S. Bimbaum, Environ. Health Perspect. 103, 157 (1995).

29 R. Neubert, R. Stahlmann, M. Korte, H. van Loveren, J. G. Vos, G. Golor, J. R. Webb, H. Helge, D. Neubert, Ann. NY. Acad. Sci. 685, 662 (1993).

30 U. G. Ahlborg, R. D. Kimbrough, E. J. Yrjänheikki, Toxic. Subst. J. 12, (1992).

31 T. Colborn, Environ. Health Perspect. 102, 55 (1994).

32 G. Lucier, G. Clark, C. Hiermath, A. Tritscher, C. Sewall, J. Huff, Toxicol. Ind. Health. 9, 631 (1993).

33 R. J. Kociba, D. G. Keyes, J. E. Beyer, R. M. Carreon, C. E. Wade, D. Dittenber, R. Kalnins, L. Frauson, C. N. Park, S. Barnard, R. Hummel, C. G. Humiston, Toxicol. Appl. Pharmacol. 46, 279 (1978).

34 G. W. Lucier, A. Tritscher, T. Goldsworthy, J. Foley, G. Clark, J. Goldstein, R. Maronpot, Cancer Res. 51, 1391 (1991).

35 S. H. Safe, Environ. Health Perspect. 103, 346 (1995).

36 J. A. Moore, J. F. Hardisty, D. A. Banas, M. A. Smith, Regul. Toxicol. Pharmacol. 20, 362 (1994).

37 R. D. Kimbrough, R. A. Squire, R. E. Linder, J. D. Strandberg, Montali, V. W. Burse, J. Natl. Cancer Inst. 55, 1453 (1975).

38 J. D. Scribner, N. K. Mottet, Carcinogenesis 2, 1235 (1981).

39 U.S.National Cancer Institute, U.S. Department of Health, Education, and Welfare, National Institutes of Health, Bethesda, MD (1978).

40 E. S. Johnson, Crit. Rev. Toxicol. 21, 451 (1992).

41 R. J. Scheuplein, J. C. Bowers, Risk Analysis 15, 319 (1995).

42 H.-O. Adami, L. Lipworth, L. Titus-Ernstoff, C.-C. Hsieh, A. Hanberg, U. G. Ahlborg, J. Baron, D. Trichopoulos, Cancer Causes \& Control 6, 551 (1995).

43 M. S. Wolff, P. G. Toniolo, E. W. Lee, M. Rivera, N. Dubin, J. Natl. Cancer Inst. 85, 648 (1993).

44 N. Krieger, R. A. Hiatt, M. S. Wolff, JNCI 86, 1255 (1994).

45 L. Rylander, L. Hagmar, Scand. J. Work Environ. Health 21, 419 (1995).

46 L. E. Gray, W. R. Kelce, E. Monosson, J. S. Ostby, L. S. Birmbaum, Toxicol. Appl. Pharmacol. 131, 108 (1995).

47 D. L. Bjerke, R. E. Peterson, Toxicol. Appl. Pharmacol. 127, 241 (1994).

48 W. H. Bulger, D. Kupfer, Am. J. Ind. Med. 4, 163 (1983).

49 Y. L. Guo, T. J. Lai, S. H. Ju, Y. C. Chen, C. C. Hsu, Dioxin'93 14, 235 (1993).

50 E. D. Levin, S. L. Schantz, R. E. Bowman, Arch. Toxicol. 62, 267 (1988).

51 S. L. Schantz, E. D. Levin, R. E. Bowman, Environ. Toxicol. Chem. 10, 747 (1991).

52 S. L. Schantz, R. E. Bowman, Neurotoxicol. Teratol. 11, 13 (1989).

53 Y. L. Guo, G. H. Lambert, C. C. Hsu, Environ. Health Perspect. 103, 117 (1995).

54 J. L. Jacobson, S. W. Jacobson, H. E. B. Humphrey, J. Pediatr. 116, 38 (1990).

55 J. L. Jacobson, S. W. Jacobson, G. A. Padgett, G. A. Brumitt, R. L. Billings, Developmental Psychology 28, 297 (1992).

56 S. W. Jacobson, G. G. Fein, J. L. Jacobson, P. M. Schwartz, J. K. Dowler, Child Dev. 56, 853 (1985).

57 B. C. Gladen, W. J. Rogan, P. Hardy, J. Thullen, J. Tingelstad, M. Tully, J. Pediatr. 113, 991 (1988).

58 M. Huisman, C. Koopman-Essenboom, V. Fidler, M. Hadders-Algra, C. G. van der Paauw, L. G. M. T. Tuinstra, N. Weisglas-Kuperus, P. J. J. Sauer, B. C. L. Touwen, E. R. Boersma, Early Hum. Dev. 41, 111 (1995).

59 M. P. Holsapple, N. K. Snyder, S. C. Wood, D. L. Morris, Toxicology 69, 219 (1991).

60 W. J. Rogan, B. C. Gladen, K.-L. Hung, S.-L. Koong, L.-Y. Shih, J. S. Taylor, Y.-C. Wu, D. Yang, N. B. Ragan, C.-C. Hsu, Science 241, 334 (1988).

61 N. Weisglas-Kuperus, T. C. J. Sas, C. Koopman-Essenboom, C. W. van der Zwan, M. A. J. Deridder, A. Beishuizen, H. Hooijkaas, P. J. J. Sauer, Pediatr. Res. 38, 404 (1995).

62 L. Hagmar, T. Hallberg, M. Leja, A. Nilsson, A. Schütz, Toxicol. Lett. 77, 335 (1995). 